- Original Article

\title{
Relationship between Smoking and Abdominal Aorta Calcification on Computed Tomography
}

\author{
Jin-Gyu Jung', Li-Tzy Wu'², Jong-Sung Kim ${ }^{1, *}$, Eung-Du Kim', Seok-Joon Yoon ${ }^{1}$ \\ 'Department of Family Medicine, Chungnam National University Hospital, Daejeon, Korea \\ ${ }^{2}$ Department of Psychiatry and Behavioral Sciences, School of Medicine and Duke Clinical Research Institute, Duke University Medical Center, \\ Durham, NC, USA
}

Background: Cigarette smoking increases the risk of atherosclerosis, which often develops as vascular calcification on radiologic examinations. This study evaluated the relationship between smoking-related factors and incidental abdominal aorta calcification (AAC) detected by computed tomography (CT) among middle-aged and elderly men.

Methods: We assessed the abdominal CT findings of 218 men aged 40 to 81 years who underwent health checkups. The associations between smoking factors and AAC were analyzed using logistic regression analysis to adjust for confounding variables such as age, lifestyle factors, and chronic diseases.

Results: Adjusting for confounding variables, the risk of AAC was significantly increased in association with smoking for at least 20 years (adjusted odds ratio [AOR], 5.22; 95\% confidence interval [CI], 1.82-14.93), smoking 10+ pack-years (10-20 pack-years: AOR, 4.54; 95\% CI, 1.07-5.68; >20 pack-years: AOR, 5.28; 95\% CI, 2.10-13.31), and a history of smoking (former smoker: AOR, 2.10; 95\% CI, 1.07-5.68; current smoker: AOR, 5.05; 95\% CI, 2.08-12.26). In terms of the daily smoking amount, even a low smoking level increased the risk of AAC.

Conclusion: These findings suggest that smoking for 20+ years, smoking $10+$ pack-years, and even a low level of smoking daily increases the likelihood of developing AAC. Clinicians should recommend that patients quit smoking and stress the importance of smoking duration when promoting health in middle-aged and elderly patients.

Keywords: Smoking; Abdominal Aorta Calcification; Atherosclerosis; Computed Tomography

Received: July 27, 2017, Revised: November 10, 2017, Accepted: December 12, 2017

${ }^{*}$ Corresponding Author: Jong-Sung Kim https://orcid.org/0000-0001-5945-0784

Tel: +82-42-280-8172, Fax: +82-42-280-7879, E-mail: jskim@ @nuh.co.kr 


\section{INTRODUCTION}

Atherosclerosis produces atheromatous or fibrous fatty plaques that may detach from the vessel walls, thereby blocking the blood vessel and causing ischemic heart disease or cerebrovascular disease. ${ }^{1,2)} \mathrm{Cal}-$ cium deposits can be observed in most cases of advanced atheroma. In other words, vascular calcification implies either advanced atherosclerosis or subclinical cardiovascular disease. ${ }^{3,4)}$ Abdominal aorta calcification (AAC) and calcification of the coronary arteries and aortic arch are considered markers of subclinical atherosclerotic disease that are independent predictors of subsequent vascular morbidity and mortality. ${ }^{5}$ Wilson et al. ${ }^{6)}$ measured the amount of calcification of the abdominal aorta using lumbar radiography and reported that AAC was a marker of subclinical atherosclerotic disease and an independent predictor of subsequent vascular morbidity and mortality. Another study used multidetector-row computed tomography (CT) to quantify the calcification of the abdominal aorta and common iliac artery and found that the calcification level was associated with not only smoking history but also high blood pressure, diabetes, chronic kidney disease, and coronary artery disease. ${ }^{7)}$ Lehmann et al. ${ }^{8)}$ found that middle-aged men who smoke developed coronary artery calcification 10 years earlier than never-smokers. This time span was 8 years in middle-aged women who smoke.

Regular or chronic smoking increases the heart rate and blood pressure, reflecting persistent stimulation of the sympathetic nervous system. Smoking-induced oxidative stress also contributes to several mechanisms of cardiovascular disease, including inflammation, endothelial dysfunction, and lipid abnormalities (e.g., oxidation of lowdensity lipoprotein and platelet activation. ${ }^{9)}$ Radiologic studies on the association of smoking with vascular calcification have shown that smoking for a high number of pack-years contributes to coronary artery and aortic calcium deposition and that middle-aged men who smoke develop coronary artery calcification 10 years earlier than never-smokers. ${ }^{8,10)}$ These studies focused mainly on coronary artery or thoracic aortic calcification.

Abdominal CT is commonly used in the clinical setting. AAC is usually asymptomatic and commonly detected on CT even in healthy people, because this modality is the most sensitive tool with which to identify vascular calcification. Lesion-focused CT tends to result in overlooking AAC in the clinical setting. It is necessary to assess potent risk factors associated with atherosclerosis, including smoking-related factors such as smoking duration (years), amount, and status and incidental AAC during health check-ups. This study examined the association between smoking and AAC detected by CT in asymptomatic Korean middle-aged and elderly men.

\section{METHODS}

\section{Study Subjects}

Among 13,432 patients who visited the health promotion center of Chungnam National University Hospital in Daejeon between January
1, 2009, and December 31, 2010. We screened 238 men aged 40 to 81 years who underwent health check-ups that included abdominal CT. Of these, 20 patients with missing data on lifestyle factors were excluded. In other words, the exclusion criteria were as follows: nonperformance of abdominal CT, female sex, cardiovascular disease history, or missing data. Ultimately, 218 patients were enrolled, and their medical records and abdominal CT results were retrospectively reviewed. This study was approved by the Institutional Review Board of National University Hospital (IRB approval no., 2015-12-015). Informed consent was waived.

\section{Data Collection}

Data on all patients were collected from health check-up records. Factors that were considered to be associated with atherosclerosis, including age, body mass index (BMI), waist circumference, systolic blood pressure (SBP) and diastolic blood pressure (DBP), total cholesterol, triglyceride (TG), high-density lipoprotein cholesterol (HDL-C), and low-density lipoprotein cholesterol (LDL-C), were abstracted for the data analysis,. The patients were divided into three smoking categories as recommended by the US Centers for Disease Control and Prevention: ${ }^{11)}$ current, former, and never-smokers.

Patients who reported smoking at least 100 cigarettes in their lifetime and, at the time of the survey, smoked either every day or on some days were defined as current smokers. Patients who reported smoking at least 100 cigarettes in their lifetime and, at the time of the survey, did not smoke at all were classified as former smokers. Patients who reported never having smoked 100 cigarettes were classified as never-smokers. The exercise status of the patients was classified using the American Heart Association recommendations: ${ }^{12)}$ those who did not exercise (no exercise group), those who exercised less than three times per week (irregular exercise group), and those who exercised three times or more per week for at least 30 minutes per session (regular exercise group). The weekly amount of alcohol consumed was calculated from the drinking frequency per week and drinks per day. To adjust for chronic medical diseases in the analysis, diabetes, hypertension, and dyslipidemia were assessed. SBP and DBP were measured in the sitting position using an automatic sphygmomanometer (BP-203 RV III; OMRON Matsusaka Co. Ltd., Matsusaka, Japan). Patients were identified as having hypertension if they were taking antihypertensive drugs or had a measured SBP of $\geq 140 \mathrm{~mm} \mathrm{Hg}$ or DBP of $\geq 90 \mathrm{~mm} \mathrm{Hg}$. We defined diabetes as a fasting blood sugar level of $\geq 126 \mathrm{mg} / \mathrm{dL}$ or the use of antidiabetic medication. Dyslipidemia was defined as receiving treatments for dyslipidemia based on the recommendation of National Cholesterol Education Program's Adult Treatment Panel III guideline for LDL or a total cholesterol level $\geq 240 \mathrm{mg} / \mathrm{dL}$ or TG level $\geq 150 \mathrm{mg} / \mathrm{dL}$.

\section{Abdominal Aorta Calcification Measurement}

To evaluate the level of AAC and common iliac artery calcification, multidetector row CT (Brilliance 64; Philips, Amsterdam, The Netherlands) images were analyzed. The overall severity of calcification was 
graded semiquantitatively using a 5-point scale, ${ }^{13)}$ ranging from absent or minimal calcifications to severe diffuse calcifications using the following criteria: (1) grade 1, either (a) no arterial calcifications; (2) grade 2 , mild noncircumferential noncontiguous arterial calcifications involving numerous segments with $<50 \%$ involvement of individual segments; (3) grade 3, moderate noncircumferential noncontiguous calcifications of multiple arterial segments with $>50 \%$ calcification of multiple segments; (4) grade 4, moderate calcifications involving multiple segments of arteries with most areas having $>50 \%$ involvement with calcification and the presence or absence of isolated completely concentric calcifications; and (5) grade 5, diffuse calcifications with multiple levels of completely concentric calcifications.

A single physician assessed the lesions to avoid individual bias. We divided the patients into two groups, those with no calcification (grade 1) and those with calcification (grades 2-5), because the frequencies of grades 3 to 5 were too small to evaluate the relationship between AAC and smoking-related variables.

\section{Statistical Analysis}

We compared the characteristics of the patients according to the smoking status. The chi-square test was used to compare categorical variables, and one-way analysis of variance with Scheffe's post hoc test was used to compare age; BMI; waist circumference; SBP; DBP; total cholesterol, HDL-C, LDL-C, and TG levels; exercise time; and weekly drinking quantity. The percentages of patients with diabetes, hypertension, dyslipidemia, and AAC among the three groups were compared using the chi-square test. Logistic regression analysis, adjusting for confounding variables, was used to assess the effects of smokingrelated factors on AAC. All statistical analyses were conducted using IBM SPSS ver. 20.0 (IBM Corp., Armonk, NY, USA). The level of statistical significance was set at $\mathrm{P}$-value $<0.05$.

\section{RESULTS}

\section{General Characteristics of the Patients}

The average age of former smokers was significantly higher than that of never-smokers and current smokers $(\mathrm{P}<0.05)$. However, there was no significant difference in BMI, waist circumference, HDL-C level, SBP, or DBP among the three groups. Total cholesterol and LDL-C levels of current smokers were significantly lower than those in neversmokers and former smokers $(\mathrm{P}<0.05)$. TG levels were lower in former smokers than those in never-smokers and current smokers $(\mathrm{P}<0.01)$. The weekly exercise frequency was lower in current smokers than that in never-smokers and former smokers $(\mathrm{P}<0.01)$, and the exercise pattern showed a different distribution among the three groups $(\mathrm{P}<0.01)$. With respect to medical diseases that might affect atherosclerosis, there were no significant differences in the proportion of patients with hypertension. The proportion of patients with diabetes and dyslipidemia was significantly different among the three groups $(\mathrm{P}<0.01)$. AAC was found in $37.6 \%$ of the study sample (Table 1 ).

\section{Smoking and Abdominal Aorta Calcification}

There were significant differences in the distribution of AAC according to all smoking-related variables, including smoking status, smoking

Table 1. Characteristics of the patients $(n=218)$

\begin{tabular}{|c|c|c|c|}
\hline Characteristic & Never-smokers $(n=94)$ & Former smokers $(n=54)$ & Current smokers $(n=70)$ \\
\hline Age $(y)$ & $53.7 \pm 10.2$ & $58.3 \pm 9.3^{*}$ & $54.8 \pm 9.4$ \\
\hline Body mass index (kg/m²) & $25.4 \pm 2.7$ & $25.1 \pm 2.6$ & $25.3 \pm 3.1$ \\
\hline Waist circumference (cm) & $87.9 \pm 8.2$ & $87.7 \pm 7.5$ & $87.7 \pm 8.8$ \\
\hline \multicolumn{4}{|l|}{ Exercise pattern ${ }^{\dagger}$} \\
\hline None & $24(25.5)$ & $16(29.6)$ & $23(32.9)$ \\
\hline Irregular & $41(43.6)$ & $15(27.8)$ & $31(44.3)$ \\
\hline Regular & $29(30.9)$ & $23(42.6)$ & $16(22.9)$ \\
\hline Weekly exercise time (min) & $102.5 \pm 95.6$ & $113.8 \pm 103.8$ & $80.0 \pm 92.1^{\dagger}$ \\
\hline Systolic blood pressure (mm Hg) & $131.7 \pm 13.7$ & $130.4 \pm 10.9$ & $130.0 \pm 16.5$ \\
\hline Diastolic blood pressure (mm Hg) & $82.9 \pm 10.4$ & $80.4 \pm 9.2$ & $81.7 \pm 12.6$ \\
\hline Total cholesterol level (mg/dL) & $192.7 \pm 31.5$ & $192.0 \pm 25.3$ & $188.2 \pm 39.2^{*}$ \\
\hline High-density lipoprotein cholesterol level (mg/dL) & $47.9 \pm 9.9$ & $45.4 \pm 8.1$ & $46.5 \pm 7.9$ \\
\hline Low-density lipoprotein cholesterol level (mg/dL) & $111.7 \pm 27.8$ & $113.4 \pm 21.7$ & $107.4 \pm 32.6^{*}$ \\
\hline Triglyceride level $(\mathrm{mg} / \mathrm{dL})$ & $167.1 \pm 106.3$ & $141.2 \pm 65.0^{\dagger}$ & $178.4 \pm 132.7$ \\
\hline Drinks/wk & $5.9 \pm 4.7$ & $6.6 \pm 12.5$ & $7.3 \pm 8.6$ \\
\hline Diabetes mellitus $^{\dagger}$ & $13(13.8)$ & $16(29.6)$ & $17(24.3)$ \\
\hline Hypertension & $43(45.7)$ & $28(51.9)$ & $34(48.6)$ \\
\hline Dyslipidemia $^{\dagger}$ & $33(35.1)$ & $27(50.0)$ & $36(51.4)$ \\
\hline Smoking duration (y) & $0^{\dagger}$ & $14.3 \pm 9.9$ & $27.1 \pm 10.5$ \\
\hline Amount smoked daily (packs/d) & $0^{\dagger}$ & $0.8 \pm 0.4$ & $0.9 \pm 0.5$ \\
\hline Amount smoked (pack-years) & $0^{\dagger}$ & $0.9 \pm 0.5$ & $24.4 \pm 6.25$ \\
\hline
\end{tabular}

Values are presented as mean \pm standard deviation or number (\%). Diabetes mellitus: fasting blood sugar level of $\geq 126 \mathrm{mg} / \mathrm{dL}$ or the use of antidiabetic medication. Hypertension: hypertension treatments or if the systolic blood pressure was $\geq 140 \mathrm{~mm} \mathrm{Hg}$ or diastolic blood pressure was $\geq 90 \mathrm{~mm} \mathrm{Hg}$. Dyslipidemia: treatments for dyslipidemia or the total cholesterol level was $\geq 240 \mathrm{mg} / \mathrm{dL}$ or the TG level was $\geq 200 \mathrm{mg} / \mathrm{dL}$.

${ }^{*} \mathrm{P}<0.05,{ }^{\mathrm{T}} \mathrm{P}<0.01$ compared with the other groups according to one-way analysis of variance with Scheffe's post hoc test or chi-square test. 
duration (years), daily smoking amount (packs/d), and overall smoking amount (pack-years). The percentages of patients with AAC among never-smokers, former smokers, and current smokers were $22.3 \%$, $44.4 \%$, and $52.9 \%$, respectively $(\mathrm{P}<0.001)$. With respect to smoking duration (years), the percentage of patients with AAC among those who had smoked for $\geq 20$ years was $57.8 \%$, which was significant different from the percentages among never-smokers and those who had smoked for $<20$ years $(\mathrm{P}<0.001)$. With respect to the daily smoking amount, the proportion of patients with AAC was significantly different among the groups (never-smokers, $22.3 \%$; $<0.5$ pack/d, 36.6\%; 0.51 pack/d, 54.4\%; and $>1$ pack/d, 60.0\%; $\mathrm{P}<0.001$ ). The proportion of patients with AAC was also significantly different according to the smoking amount (pack-years) $(\mathrm{P}<0.001)$ (Table 2).

\section{Odds Ratio of Abdominal Aorta Calcification Based on Smoking Characteristics}

To evaluate the factors associated with AAC, we performed logistic regression analyses adjusting for confounding variables such as age; $\mathrm{ex}$ ercise; waist circumference; SBP, DBP, HDL-C, LDL-C, and TG levels; diabetes; hypertension; and dyslipidemia. Regarding the smoking period, smoking for $\geq 20$ years compared with never smoking was associated with an increased adjusted odds ratio (AOR) of having AAC (AOR, 5.22; 95\% confidence interval [CI], 1.82-14.93). Other smoking-related variables associated with AAC were 10+ pack-years of smoking (10-20 pack-years: AOR, 4.54; 95\% CI, 1.47-14.03; >20 pack-years: AOR, 5.28; 95\% CI, 2.10-13.31), smoking history (former smoker: AOR, 2.10; 95\% CI, 1.07-5.68; current smoker: AOR, 5.05; 95\% CI, 2.08-12.26), daily smoking amount (<0.5 pack/d: AOR, 3.41; 95\% CI, 1.15-10.11; 0.5-1 pack/d: AOR, 3.74; 95\% CI, 1.51-9.29; >1 pack/d: AOR, 6.11; 95\% CI,

Table 2. Abdominal aortic calcification according to smoking-related variables in all patients $(n=218)$

\begin{tabular}{cc}
\hline \multicolumn{1}{c}{ Smoking variable } & Abdominal aortic calcification \\
\hline Smoking status* & \\
Never-smoker & $22.3(21 / 94)$ \\
Former smoker & $44.4(24 / 54)$ \\
Current smoker & $52.9(37 / 70)$ \\
Smoking duration (y) ${ }^{*}$ & \\
Never-smoker & $22.3(21 / 94)$ \\
$<20$ & $26.4(14 / 53)$ \\
$\geq 20$ & $57.3(47 / 71)$ \\
Amount smoked daily (packs/d) & \\
Never-smoker & $22.3(21 / 94)$ \\
$<0.5$ & $36.6(15 / 41)$ \\
$0.5-1$ & $54.4(37 / 68)$ \\
$>1$ & $60.0(9 / 15)$ \\
Smoking amount (pack-years) ${ }^{*}$ & \\
Never-smoker & $22.3(21 / 94)$ \\
$<10$ & $20.7(6 / 29)$ \\
$10-20$ & $38.9(14 / 36)$ \\
$>20$ & $69.5(41 / 59)$ \\
\hline
\end{tabular}

Values are presented as \% (number/total number).

${ }^{*} \mathrm{P}<0.001$ by chi-square test.
1.50-24.93) (Table 3).

\section{DISCUSSION}

This study found an association between cigarette smoking and AAC among Korean middle-aged and elderly men. Smoking for more than 20 years, former or current smoking, smoking for 10+ pack-years, and even light smoking increased the odds of AAC. Cardiovascular disease develops 2 to 4 times more often in smokers, ${ }^{8,14)}$ and the risk of peripheral arterial disease is markedly elevated in smokers. ${ }^{15)}$ In this study, the AOR of AAC for current smokers was 5.0, which is in line with those in previous studies. ${ }^{10)}$ The AOR of AAC for former smokers was 2.1, which supports the finding that the risk of coronary heart disease is less than half of that in continuing smokers. ${ }^{11)}$ Additionally, although there is no statistical significance, the risk for AAC increased by $80 \%$ or more in less than 20 pack-years and $49 \%$ in less than 10 pack-years. It suggested that further studies are needed to prove that small amount and short duration of smoking have negative vascular effects such as atherosclerosis.

In particular, smoking for more than 20 years increased the risk of having AAC. Until now, no study has reported a cutoff period at which smoking increases the odds of AAC. However, our results are acceptable when the following points are considered. First, most Korean smokers started smoking in their late teens or early twenties; therefore, those who have smoked for more than 20 years would be in their late thirties or older. ${ }^{16)}$ The prevalence of metabolic syndrome, which can exacerbate atherosclerosis, begins to increase in this age group. ${ }^{17)}$ Second, atherosclerosis is a sign of aging that progresses slowly for a long

Table 3. Logistic regression analysis of abdominal aortic calcification according to smoking-related variables $(n=218)$

\begin{tabular}{lc}
\hline \multicolumn{1}{c}{ Smoking variable } & $\begin{array}{c}\text { Adjusted odds ratio* } \\
\text { (95\% confidence interval) }\end{array}$ \\
\hline Smoking status & 1.00 \\
Never-smoker & $2.10(1.07-5.68)$ \\
Former smoker & $5.05(2.08-12.26)$ \\
Current smoker & \\
Smoking duration (y) & 1.00 \\
Never-smoker & $1.81(0.30-10.95)$ \\
$<10$ & $1.95(0.67-5.81)$ \\
10 to $<20$ & $5.22(1.82-14.93)$ \\
$\geq 20$ & \\
Smoking amount (pack-years) & 1.00 \\
Never-smoker & $1.49(0.41-5.37)$ \\
$<10$ & $4.54(1.47-14.03)$ \\
$10-20$ & $5.28(2.10-13.31)$ \\
$>20$ & \\
Daily smoking amount (packs/d) & 1.00 \\
Never-smoker & \\
$<0.5$ & $3.41(1.15-10.11)$ \\
$0.5-1$ & $3.74(1.51-9.29)$ \\
$>1$ & \\
\hline
\end{tabular}

${ }^{\star}$ Adjusted for age, exercise, waist circumference, hypertension, dyslipidemia, and diabetes. 
period of time, ${ }^{1,18)}$ smoking is a potent factor that promotes atherosclerosis, ${ }^{14,15,19)}$ and vascular calcification such as that in the abdominal aorta. ${ }^{15)}$ Although autopsy studies have found more extensive atherosclerotic changes in young adults, vascular calcification tends to be detected later radiologically than pathologically. ${ }^{20,21)}$ Therefore, our findings suggest that clinicians should provide intensive counseling to middle-aged and older patients engaged in longer-term or active smoking regarding smoking and health issues, including AAC, to prevent cardiovascular complications.

An increased number of pack-years tended to increase the AOR of AAC markedly (10-20 years: AOR, 4.54; >20 years: AOR, 5.28). A study targeting middle aged Japanese and Korean found that OR of aortic calcification for smokers with higher pack-years were 3.6 in Koreans. ${ }^{10)}$ The AOR in our study was higher than that. This may be because the average age of the patients in our study was older ( $>55$ years), indicating that smoking at an older age may have a synergic effect with other cardiovascular risk factors. Although the two studies differed in their design and methods, the difference in the AOR between the two studies supports the benefits of early smoking cessation.

With respect to the daily smoking amount, the risk of AAC increased with the number of packs smoked per day, starting at $<0.5 \mathrm{pack} / \mathrm{d}$. The daily smoking amount and pack-years were associated with AAC or coronary artery calcification, which also concurs with previous studies. ${ }^{15,22)}$ These studies showed that smoking is a potent risk factor for developing cardiovascular and peripheral artery diseases. Additionally, the risk associated with smoking was striking and increased with the number of cigarettes smoked per day and number of years of smoking. ${ }^{23,24)}$ Accordingly, physicians should emphasize absolute abstinence from smoking, rather than light or moderate smoking, to their patients. Even when a patient's chief complaint is not associated with cardiovascular symptoms, smoking cessation is advised, because continued smoking may increase the likelihood of cardiovascular events.

There are some limitations to our study. First, this was a cross-sectional study and thus precludes causal associations of AAC with lifestyle factors and medical diseases. Second, this study targeted only Korean men, and all patients in this study were Korean men and has higher smoking rate than the general population. According to the 2013 Korea National Health and Nutrition Examination Survey, ${ }^{25)}$ the smoking rates among Korean women (5.4\%) were too low to evaluate any relationship between smoking and AAC. Third, we did not evaluate family history of cardiovascular disease and nutrition and which are potential factor in cardiovascular disease, and socioeconomic factors such as income and education were also not assessed. Fourth, we conducted this study only based on the questionnaire that is easily underreported to assess smoking status and amount of participants. Fifth, selection bias exists in this study because we were confined to only male subjects with abdominal CT data. Finally, semiquantitative measurement used to assess AAC was also a major limitation in this study.

Despite these limitations, this study showed that the presence of AAC detected by CT was associated with not only the smoking amount, including the daily amount and pack-years, but also smoking for $\geq 20$ years. Considering that the patients in this study were relatively healthy since we excluded patients with a cardiovascular history, it is important for clinicians to assess smoking behavior and emphasize early cessation of smoking for the promotion of health regardless of age, even in asymptomatic and healthy patients.

\section{CONFLICT OF INTEREST}

No potential conflict of interest relevant to this article was reported.

\section{ORCID}

Jin-Gyu Jung: https://orcid.org/0000-0002-2591-4867

Li-Tzy Wu: https://orcid.org/0000-0001-9724-4295

Jong-Sung Kim: https://orcid.org/0000-0001-5945-0784

Eung-Du Kim : https://orcid.org/0000-0002-9271-8540

Seok-Joon Yoon: https://orcid.org/0000-0002-6784-8191

\section{REFERENCES}

1. Badimon L, Vilahur G. Thrombosis formation on atherosclerotic lesions and plaque rupture. J Intern Med 2014;276:618-32.

2. Wexler L, Brundage B, Crouse J, Detrano R, Fuster V, Maddahi J, et al. Coronary artery calcification: pathophysiology, epidemiology, imaging methods, and clinical implications: a statement for health professionals from the American Heart Association. Writing Group. Circulation 1996;94:1175-92.

3. Rumberger JA, Simons DB, Fitzpatrick LA, Sheedy PF, Schwartz RS. Coronary artery calcium area by electron-beam computed tomography and coronary atherosclerotic plaque area: a histopathologic correlative study. Circulation 1995;92:2157-62.

4. Simon A, Giral P, Levenson J. Extracoronary atherosclerotic plaque at multiple sites and total coronary calcification deposit in asymptomatic men: association with coronary risk profile. Circulation 1995;92:141421.

5. Criqui MH, Denenberg JO, McClelland RL, Allison MA, Ix JH, Guerci A, et al. Abdominal aortic calcium, coronary artery calcium, and cardiovascular morbidity and mortality in the Multi-Ethnic Study of Atherosclerosis. Arterioscler Thromb Vasc Biol 2014;34:1574-9.

6. Wilson PW, Kauppila LI, O'Donnell CJ, Kiel DP, Hannan M, Polak JM, et al. Abdominal aortic calcific deposits are an important predictor of vascular morbidity and mortality. Circulation 2001;103:1529-34.

7. Sun WY, Jang LC. Calcification in the abdominal aorta and common iliac artery. J Korean Soc Vasc Surg 2008;24:94-100.

8. Lehmann N, Mohlenkamp S, Mahabadi AA, Schmermund A, Roggenbuck U, Seibel R, et al. Effect of smoking and other traditional risk factors on the onset of coronary artery calcification: results of the Heinz Nixdorf recall study. Atherosclerosis 2014;232:339-45.

9. Chobanian AV, Bakris GL, Black HR, Cushman WC, Green LA, Izzo JL Jr, et al. The seventh report of the joint National Committee on Prevention, Detection, Evaluation, and Treatment of High Blood Pressure: the JNC 7 report. JAMA 2003;289:2560-72. 
10. Hirooka N, Kadowaki T, Sekikawa A, Ueshima H, Choo J, Miura K, et al. Influence of cigarette smoking on coronary artery and aortic calcium among random samples from populations of middle-aged Japanese and Korean men. J Epidemiol Community Health 2013;67:11924.

11. Centers for Disease Control and Prevention, National Center for Chronic Disease Prevention and Health Promotion, Office on Smoking and Health. How tobacco smoke causes disease: the biology and behavioral basis for smoking-attributable disease: a report of the surgeon general. Atlanta (GA): Centers for Disease Control and Prevention; 2010.

12. Fletcher GF, Balady G, Blair SN, Blumenthal J, Caspersen C, Chaitman $\mathrm{B}$, et al. Statement on exercise: benefits and recommendations for physical activity programs for all Americans: a statement for health professionals by the Committee on Exercise and Cardiac Rehabilitation of the Council on Clinical Cardiology, American Heart Association. Circulation 1996;94:857-62.

13. Lockhart ME, Robbin ML, McNamara MM, Allon M. Association of pelvic arterial calcification with arteriovenous thigh graft failure in haemodialysis patients. Nephrol Dial Transplant 2004;19:2564-9.

14. Patel SA, Winkel M, Ali MK, Narayan KM, Mehta NK. Cardiovascular mortality associated with 5 leading risk factors: national and state preventable fractions estimated from survey data. Ann Intern Med 2015;163:245-53.

15. Lu JT, Creager MA. The relationship of cigarette smoking to peripheral arterial disease. Rev Cardiovasc Med 2004;5:189-93.

16. Kim ED, Kim JS, Kim SS, Jung JG, Yun SJ, Kim JY, et al. Association of abdominal aortic calcification with lifestyle and risk factors of cardiovascular disease. Korean J Fam Med 2013;34:213-20.

17. Bullen C. Impact of tobacco smoking and smoking cessation on car- diovascular risk and disease. Expert Rev Cardiovasc Ther 2008;6:88395.

18. Lee YH, Shin MH, Kweon SS, Choi JS, Rhee JA, Ahn HR, et al. Cumulative smoking exposure, duration of smoking cessation, and peripheral arterial disease in middle-aged and older Korean men. BMC Public Health 2011;11:94.

19. Wong ND, Lopez VA, Allison M, Detrano RC, Blumenthal RS, Folsom AR, et al. Abdominal aortic calcium and multi-site atherosclerosis: the Multiethnic Study of Atherosclerosis. Atherosclerosis 2011;214:436-41.

20. McGill HC Jr, McMahan CA, Malcom GT, Oalmann MC, Strong JP. Effects of serum lipoproteins and smoking on atherosclerosis in young men and women. The PDAY Research Group. Pathobiological Determinants of Atherosclerosis in Youth. Arterioscler Thromb Vasc Biol 1997;17:95-106.

21. McGill HC Jr, McMahan CA, Gidding SS. Preventing heart disease in the 21st century: implications of the Pathobiological Determinants of Atherosclerosis in Youth (PDAY) study. Circulation 2008;117:1216-27.

22. Lee YA, Kang SG, Song SW, Rho JS, Kim EK. Association between metabolic syndrome, smoking status and coronary artery calcification. PLoS One 2015;10:e0122430.

23. El-Saed A, Curb JD, Kadowaki T, Okamura T, Sutton-Tyrrell K, Masaki $\mathrm{K}$, et al. The prevalence of aortic calcification in Japanese compared to white and Japanese-American middle-aged men is confounded by the amount of cigarette smoking. Int J Cardiol 2013;167:134-9.

24. Kumagai S, Amano T, Takashima H, Waseda K, Kurita A, Ando H, et al. Impact of cigarette smoking on coronary plaque composition. Coron Artery Dis 2015;26:60-5.

25. Korea Centers for Disease Control and Prevention. The sixth Korea National Health and Nutrition Examination Survey (KNHANES III). Cheongju: Korea Centers for Disease Control and Prevention; 2014. 